Revista de Psicología Vol. 39 (2), 2021 (e-ISSN 2223-3733)

\title{
Impacto do Funcionamento Familiar na Diferenciaçáo do Self de Universitários Brasileiros
}

\author{
Milena Carolina Fiorini ${ }^{1}$, Marúcia Patta Bardagi², Luciane Guisso ${ }^{3}$, \\ Maria Aparecida Crepaldi ${ }^{4}$ \\ Universidade Federal de Santa Catarina-Brasil
}

\begin{abstract}
A relação entre funcionamento familiar e diferenciação do self é consolidada por autores sistêmicos clássicos, porém pouco explorada por pesquisas empíricas, especialmente no Brasil. Este estudo quantitativo teve como objetivo analisar as relaçôes entre funcionamento familiar e diferenciação do self de universitários brasileiros. Participaram da pesquisa 800 graduandos, de ambos os sexos, com idade média de 22.7 anos. Constatou-se que graduandos com níveis mais elevados de diferenciação do self perceberam de forma mais positiva a comunicação e a satisfação familiares. As famílias emaranhadas demonstraram ser as menos favoráveis ao processo de diferenciação. Verificou-se que indivíduos do sexo masculino e graduandos mais velhos apresentaram níveis maiores de diferenciação. Por fim, universitários com índices mais elevados de diferenciação e pertencentes às famílias consideradas saudáveis mostraram-se mais satisfeitos com o curso. Esses achados podem contribuir para a atuação de psicólogos com jovens adultos universitários, seja no contexto clínico ou de aconselhamento de carreira.

Palavras-chave: diferenciação do self, constituição do self, relaçóes familiares, ambiente familiar, ambiente universitário.
\end{abstract}

1 Mestre em Psicologia. Doutoranda em Universidade Federal de Santa Catarina, Brasil. Dirección Postal: Rua Ogê Fortkamp, 111, apto 508 D, Trindade -Florianópolis/ SC-Brasil, CEP: 88 036-610. Contato: milenacf.psicologa@gmail.com. https://orcid. org/0000-0003-1390-1564

2 Mestre e doutora em Psicologia. Professora Adjunta do curso de Psicologia da Universidade Federal de Santa Catarina, Brasil. Dirección postal: Rua Estrada de Benfica, 220,r/c esquerdo, Lisboa (Portugal) 1500-094. Contato: marucia.bardagi@gmail.com. https://orcid. org/0000-0003-0224-2794

3 Mestre em Psicologia. Doutoranda em Universidade Federal de Santa Catarina, Brasil. Dirección postal: Rua Estrada de Benfica, 220,r/c esquerdo, Lisboa (Portugal) 1500-094. Contato: lucianeguisso@gmail.com. https://orcid.org/0000-0002-4846-319X

4 Mestre em psicologia y Doutora em Saúde Mental. Professora Titular da Universidade Federal de Santa Catarina, Brasil. Dirección postal: Rua Volny Martins, 115 -Casa 4, Florianópolis/SC, CEP 88037-245. Contato: maria.crepaldi@gmail.com. https://orcid. org/0000-0002-5892-7330 
Impact of Family Functioning on the Differentiation of Self of Brazilian University Students The relationship between family functioning and differentiation of self has been established by classical systemic authors, but little explored by empirical research, especially in Brazil. This quantitative study aimed to analyze the relationship between family functioning and differentiation of self of Brazilian university students. Eight hundred undergraduates of both genders participated in the research, with an average age of 22.7 years. It was found that students with higher levels of differentiation of self perceived family communication and satisfaction more positively. Tangled families proved to be the least favorable to the differentiation process. It was found that males and older undergraduate students displayed higher levels of differentiation. Finally, university students with higher levels of differentiation, who belonged to families regarded as healthy, were more satisfied with their career These findings may contribute to the practice of psychologists with young adult university students, both in clinical and career counseling settings.

Keywords: differentiation of self, constitution of self, family relationships, family environment, university environment.

\section{Impacto del Funcionamiento Familiar en la Diferenciación del Self de Universitarios Brasileńos}

La relación entre el funcionamiento familiar y la diferenciación del self es consolidada por autores sistémicos clásicos, pero poco explorada por investigaciones empíricas, especialmente en Brasil. Este estudio cuantitativo tuvo como objetivo analizar las relaciones entre el funcionamiento familiar y la diferenciación del self de universitarios brasileños. Participaron de la encuesta 800 estudiantes de ambos sexos, con edad promedio de 22.7 ańos. Se constató que los estudiantes con niveles más elevados de diferenciación percibieron de forma más positiva la comunicación y la satisfacción familiares. Las familias enmarañadas demostraron ser las menos favorables al proceso de diferenciación. Se verificó que los varones y los estudiantes mayores presentaron niveles más altos de diferenciación. Por último, universitarios con índices más elevados de diferenciación y pertenecientes a familias consideradas sanas se mostraron más satisfechos com su carrera. Estos hallazgos pueden contribuir a la práctica de psicólogos con jóvenes universitarios, ya sea en el contexto clínico o de consejería vocacional. Palabras clave: diferenciación del self, constitución del self, relaciones familiares, ambiente familiar, ambiente universitario.

\section{Impact du fonctionnement de la famille sur la différenciation du soi des étudiants uni- versitaires brésiliens}

La relation entre le fonctionnement de la famille et l'auto-différenciation est consolidée par les auteurs systémiques classiques, mais peu explorée par les recherches empiriques, en particulier au Brésil. Cette étude quantitative visait à analyser les relations entre fonctionnement de la famille et différenciation de soi entre étudiants universitaires brésiliens. Au total, 800 étudiants de premier cycle, des deux sexes, âgés en moyenne de 22,7 ans. Il a été constaté que les étudiants de premier cycle ayant des niveaux plus élevés d'auto-différenciation percevaient la communication et la satisfaction familiales de manière plus positive. Les familles enchevêtrées se sont révélées être les moins favorables au processus de différenciation. Il a été constaté que les garçons et les élèves plus âgés avaient des niveaux de différenciation plus élevés. Enfin, les étudiants universitaires présentant des taux de différenciation plus élevés et appartenant à des familles en bonne santé étaient plus satisfaits du cours. Ces résultats peu- 
Impacto do Funcionamento Familiar na Diferenciação do Selfde Universitários Brasileiros / Forini et al.

vent contribuer à la performance des psychologues ayant de jeunes étudiants universitaires, que ce soit en milieu clinique ou en orientation professionnelle.

Mots-clés: différenciation de soi, constitution de soi, relations familiales, environnement familial, environnement universitaire 
De acordo com a Teoria Familiar Sistêmica, a família é um sistema de relaçóes interdependentes entre seus membros, e destes com o seu contexto econômico, político e cultural (Cerveny, 1994). Todas as famílias passam por estágios de desenvolvimento que contribuem para o crescimento psicossocial de seus integrantes, bem como para constantes reestruturaçóes na dinâmica familiar (Carter \& McGoldrick, 1995).

Enquanto a dinâmica familiar se refere à forma como os membros se relacionam e às adaptaçóes diante das mudanças do ciclo de vida, a estrutura de cada família diz respeito às regras que permitem ou não determinados comportamentos e regulam a interação entre as pessoas (Minuchin, 1982). O funcionamento familiar, por sua vez, compreende a constante inter-relação estabelecida entre a estrutura e a dinâmica, envolvendo os rituais cultivados conjuntamente pelos integrantes, o modo como lidam com conflitos e a determinação de papéis e níveis hierárquicos (Böing, 2014).

As transições de estágios do ciclo vital geralmente demandam a reorganização do sistema familiar e o cumprimento de certas tarefas. A fase da adultez jovem, foco deste estudo, corresponde ao estágio designado "lançando5 os filhos e seguindo em frente" (Carter \& McGoldrick, 1995). Esse momento refere-se à transição para a vida adulta e é demarcado pela diferenciação do self em relação à família de origem, definida por Bowen (1978) como a capacidade para adquirir equilíbrio entre funcionamento emocional, intelectual, intimidade e autonomia nas relações.

5 O termo "lançamento" pode ser compreendido como "emancipação", tendo em vista que a tradução literal não retrata a definição de forma fidedigna. A emancipação, em consonância com literatura, denota um distanciamento que pode ser simbólico, e náo necessariamente físico. 
A transição para a vida adulta demanda como principais tarefas dos jovens adultos a constituição de novos relacionamentos íntimos, o estabelecimento de objetivos profissionais e a aquisição gradativa de independência financeira/emocional em relação à família de origem. $\mathrm{O}$ cumprimento gradativo dessas tarefas é indicativo de um processo satisfatório de diferenciação. Já por parte dos pais, a tarefa essencial envolve a construção de uma relação menos hierárquica com os filhos, oferecendo apoio emocional/financeiro, porém permitindo que os jovens adultos adquiram sua própria autonomia, sem precisar cumprir todas as expectativas da família (Aylmer, 1995).

A adultez jovem geralmente representa, também, a saída do jovem adulto da casa dos pais para estudar, trabalhar e/ou casar, sendo que principalmente em famílias de classes média e alta, coincide com a entrada do filho na universidade. $O$ período acadêmico costuma ser perpassado pela necessidade de adaptação a um novo contexto, às mudanças nos relacionamentos e a diferentes responsabilidades por parte dos jovens adultos. Essa transiçáo pode ser geradora de angústias e até mesmo de estados depressivos por parte dos estudantes de graduação (Bardagi \& Albanaes, 2015). Além disso, a confrontação com a realidade da universidade pode gerar satisfação ou insatisfação com o curso, que, por sua vez, influencia no nível de comprometimento com as metas acadêmicas e na qualidade de vida do estudante (Bardagi \& Hutz, 2010). Alguns estudos nacionais apontam que uma das principais consequências da insatisfação com o curso é a evasão acadêmica, ou seja, a saída do universitário de seu curso de origem, sem concluí-lo (Ambiel, Hernández, \& Martins; Bardagi \& Hutz, 2010). Diante desses desafios, o funcionamento da família, que envolve a maneira como os pais e os filhos lidam com esse momento, assume papel crucial para o enfrentamento de possíveis dificuldades.

Conforme afirma Minuchin (1982), o funcionamento saudável de uma família não está ligado à ausência de problemas, mas ao seu nível de flexibilidade frente às mudanças intrínsecas ao ciclo vital. Quando se analisa a fase da adultez jovem e os desafios da vivência universitária, o funcionamento saudável da família caracteriza-se pelo encorajamento 
dos pais em relação à aquisição de independência dos filhos, aliado ao suporte emocional. Nesse caso, o processo de diferenciação do jovem adulto tende a ser estimulado, de modo que a separação da família pode ocorrer naturalmente, sem necessidade de rompimentos ou refúgios emocionais (Aylmer, 1995). Por outro lado, quando as famílias são emaranhadas, ou seja, não apresentam regras e limites claros, hierarquias e papéis definidos, e excesso de envolvimento emocional, podem contribuir para a manutenção da relaçáo de dependência do jovem adulto (Minuchin, 1982). Já quando a família apresenta alto grau de rigidez, a comunicação fica prejudicada, originando um padrão de desligamento entre os membros. A tendência, nesses casos, é o estabelecimento de uma relação de pseudo-independência com a família, e em casos mais extremos, pode resultar em rompimento da relação (Bowen, 1978).

Olson e Gorall (2006) desenvolveram um modelo de avaliação do funcionamento familiar denominado Modelo Circumplexo do Sistema Conjugal e Familiar. Esse modelo é condizente com as premissas de Minuchin (1982) e classifica o padrão de funcionamento da família em seis categorias, desde a mais saudável até a menos funcional: 1 . Famílias equilibradas: caracterizadas pelo equilíbrio entre proximidade emocional e flexibilidade diante de mudanças; 2 . Rigidamente equilibradas: determinadas pelo alto nível de proximidade entre os membros e disposição razoável para mudanças, porém com certa rigidez; 3. Medianas: definidas pela integração relativamente saudável entre os membros, com equilíbrio entre todas as dimensóes; 4. Flexivelmente equilibradas: denota baixa coesão entre os membros, porém com alto grau de flexibilidade; 5. Caoticamente desligadas: apresentam falta de proximidade emocional e dificuldade para lidar com mudanças; e 6. Desequilibradas: possuem menor nível de funcionalidade, devido aos altos escores em todas as dimensóes negativas.

Uma revisão de literatura internacional desenvolvida por Miller, Anderson e Keala (2004) buscou identificar pesquisas empíricas focadas na relação entre funcionamento da família e diferenciação do self. A partir da sistematização construída pelos autores, concluiu-se que a maioria dos estudos foram realizados no Oriente e têm legitimado a 
relação positiva entre funcionamento familiar saudável e elevados níveis de diferenciação (Lee \& Cha, 2000; Lee \& Lee, 1997). Outras pesquisas posteriores, também orientais, vêm legitimando esses achados (Chung \& Gale, 2008; Kim et al., 2014; Kim et al., 2015). A única investigação ocidental encontrada (Johnson, Thorngren \& Smith, 2001) foi desenvolvida nos EUA, e os resultados sugeriram que jovens adultos de famílias divorciadas apresentavam altos níveis de corte emocional e reatividade emocional (dimensóes negativas da diferenciação do self).

No cenário brasileiro, tanto o construto diferenciação do self quanto o funcionamento familiar ainda são pouco explorados cientificamente. Foram identificadas apenas quatro pesquisas nacionais, todas qualitativas, que relacionam as duas temáticas. Um dos estudos foi realizado por Reis e Rabinovich (2006), com jovens adultas baianas de baixa renda. Os resultados indicaram que a rigidez existente na relação entre mães e filhas, aliada ao fato de as jovens mulheres assumirem diversas responsabilidades que caberiam aos pais prejudicavam o seu processo de diferenciaçáo, de modo que uma das principais consequências era o abandono dos estudos. Duas pesquisas investigaram o impacto da diferenciação do self na união conjugal de jovens adultos (Bueno, Souza, Monteiro, \& Teixeira, 2013; Martins, Rabinovich, \& Silva, 2008). Os achados foram consoantes no sentido de considerar que a dificuldade de diferenciação de self de um ou de ambos os parceiros em relação às suas famílias de origem tende a reproduzir padróes repetitivos de funcionamento que geram problemas no relacionamento conjugal. O estudo de caso desenvolvido por Pellegrini, Silva, Barreto e Crepaldi (2015) relatou o processo de atendimento familiar de um jovem adulto e sua mãe, cuja demanda inicial era o excesso de conflitos entre os dois. As autoras identificaram que o padráo de funcionamento da família era demarcado pelo desligamento emocional entre os pais e pelo emaranhamento entre mãe e filho, que conjuntamente, acabavam por dificultar a diferenciação do self do jovem adulto.

Com base no contexto apresentado, o objetivo deste estudo foi analisar as relaçôes entre funcionamento familiar e nível de diferenciação do self de jovens adultos universitários brasileiros. Como objetivos 
secundários, buscou-se identificar diferenças de sexo, faixa etária e nível de satisfação com o curso de graduação dos universitários em relação aos níveis de funcionamento familiar e diferenciação do self. A relevância desta pesquisa pauta-se na possibilidade de encontrar subsídios para a estruturação de estratégias/intervenções no contexto clínico de atendimento psicológico individual/familiar de jovens adultos graduandos, bem como em processos de aconselhamento de carreira e gestão universitária.

\section{Método}

\section{Participantes}

O estudo teve como público-alvo 800 estudantes universitários, de 70 diferentes cursos de graduação de universidades públicas e privadas do Brasil, com idades entre 18 e 35 anos $(M=22.7$; $\mathrm{DP}=3.92)$ e de ambos os sexos. Houve predomínio de participantes da região sul $(87.4 \%)$ e do sexo feminino $(66 \%)$.

\section{Medição}

a) Questionário sociodemográfico: abarcou perguntas sobre dados pessoais, acadêmicos e familiares, além de uma questão de múltipla escolha para avaliar o nível de satisfação com o curso, com as seguintes opçóes: 1 Insatisfeito; 2. Pouco satisfeito; 3. Satisfeito; e 4. Muito satisfeito.

b) Escala de Avaliação da Flexibilidade e Coesão Familiar (FACES IV - Family Adaptability and Cohesion Evaluation Scales) (Olson \& Gorall, 2006; Olson, Gorall, \& Tiesel, 2007; traduzido para o Brasil por Minetto, 2010): escala de autorrelato composta por 62 itens, avalia o funcionamento familiar por meio de quatro dimensóes: 1. Coesão: diz respeito à ligação emocional entre os membros; 2. Flexibilidade: refere-se à capacidade da família para alterar suas regras, sua estrutura de poder e os papéis dos membros, diante de situaçóes de mudança e/ou crise; 3. Comunicação: reflete a habilidade dos membros para 
comunicarem-se de forma positiva e; 4. Satisfação: relaciona-se à forma como cada membro se sente enquanto parte da família.

O resultado do padrão de funcionamento familiar é obtido a partir da relação entre as dimensóes coesão e flexibilidade, gerada por meio de seis níveis de resultados, dois positivos, referentes à coesão equilibrada e à flexibilidade equilibrada; e quatro negativos, que se referem à coesão desengajada (indica baixa proximidade emocional e alto nível de independência); coesão emaranhada (denota alta proximidade e dependência excessiva); flexibilidade rígida (caracteriza-se por rigorosidade na disciplina familiar e delimitação rígida de papéis e funções, aliadas à dificuldade para lidar com mudanças); e flexibilidade caótica (sugere falta de disciplina e ausência de delimitação clara de papéis e funções) (Olson et al., 2007).

As dimensões da comunicação familiar e satisfação familiar são interpretadas de forma mais simplificada, a partir da soma dos itens para obtenção de um escore geral (Olson \& Gorall, 2006). Os índices de consistência interna da FACES IV foram considerados entre bons ou excelentes, sendo neste estudo identificados: escala total: .90; coesão equilibrada: .85; flexibilidade equilibrada: .81; coesão desengajada: .81; coesão emaranhada: .61; flexibilidade rígida: .75; e flexibilidade caótica: .78.

c) Inventário de Diferenciação do SelfRevisto (DSI-R - Differentiation of Self Inventory Revised) (Skowron \& Friedlander, 1998; validado para Portugal por Major, Rodriguez-Gonzalez, Miranda, Rousselot \& Relvas, 2014): instrumento de autorrelato que mensura a diferenciaçáo do self, por meio de perguntas sobre as relaçóes significativas do sujeito com a família e outras pessoas de seu cotidiano. O questionário contém 46 itens, divididos em quatro dimensóes: 1. Reatividade emocional: avalia a capacidade para responder aos estímulos ambientais com base em respostas emocionais automáticas; 2 . Corte emocional: denota o limite/distanciamento emocional e comportamental em relação aos outros, além de um possível temor em relação à intimidade nas relações íntimas; 3. Posição do eu: caracteriza-se por um sentido claro e definido do self, que confere capacidade para manter as crenças e convicçóes 
pessoais mesmo quando pressionado a fazer o contrário; e 4. Fusão com outros: refere-se à identificação e ao envolvimento emocional com outras pessoas, e, quando elevada, pode gerar certa dependência afetiva (Skowron \& Schmitt, 2003). Neste estudo, os índices de consistência interna foram apropriados: escala total: .90; reatividade emocional: .85; posição do eu: .78; corte emocional: .76; e fusão com os outros: .76.

\section{Procedimentos}

Os instrumentos foram aplicados de forma online, por meio de um protocolo desenvolvido na plataforma Google Docs. Os participantes foram contatados via redes sociais, fóruns de graduação, centros acadêmicos e coordenadorias dos cursos de graduação.

Todos os procedimentos empregados na pesquisa seguiram a Resolução 466/2012 (Conselho Nacional de Saúde, 2012), que trata das regras sobre pesquisa com Seres Humanos nas Ciências Humanas e Sociais. O estudo foi autorizado pelo Comitê de Ética em Pesquisa com Seres Humanos (CEPSH) da Universidade Federal de Santa Catarina (UFSC), com número de parecer 1.556.427. Anteriormente ao preenchimento dos materiais, os participantes consentiram com os termos estabelecidos no TCLE - Termo de Consentimento Livre e Esclarecido. Os interessados receberam o relatório final do estudo, via e-mail, como devolutiva da participação, que foi voluntária e resguardada pela garantia de anonimato.

\section{Análise dos dados}

A análise dos dados foi realizada com apoio do SPSS - Statistical Package of Social Sciences 22.0. O procedimento inicial consistiu na verificação dos índices de consistência interna (Alfa de Cronbach) dos instrumentos utilizados. Posteriormente, foram empregados testes de estatística descritiva (médias e desvios padrão) e inferencial, conforme características específicas de cada variável. 


\section{Resultados}

As médias e os desvios padrão da diferenciação do self e suas dimensóes, gerais e por sexo, podem ser visualizados na Tabela 1. O teste $t$ foi empregado para verificar se havia diferenças estatisticamente significativas entre as médias da diferenciação do self para homens e mulheres. Os resultados foram positivos tanto para o escore total da diferenciação $(t=5.56 ; g l=797 ; \mathrm{p}<.00)$ quanto para as dimensóes reatividade emocional $(t=8.11 ; g l=797 ; p<.00)$, posição do eu $(t=4.27$; $g l=797 ; p<.00)$ e fusão com os outros $(t=5.93 ; g l=797 ; p<.00)$, de modo que os universitários do sexo masculino obtiveram médias mais elevadas do que as mulheres. Se comparados os sexos, a única dimensão da diferenciação que não indicou diferença entre as médias foi o corte emocional $(t=2.37 ; g l=797 ; p<.08)$.

\section{Tabela 1}

Diferenciação do self: médias e desvios padrão gerais e por sexo

\begin{tabular}{lcccccc}
\hline & \multicolumn{2}{c}{ Geral } & \multicolumn{2}{c}{ Masculino } & \multicolumn{2}{c}{ Feminino } \\
\cline { 2 - 7 } & \multicolumn{1}{c}{$M$} & $D P$ & $M$ & $D P$ & $M$ & $D P$ \\
\cline { 2 - 7 } Diferenciação do Self(total) & 162.71 & 30.7 & 170.97 & 30.38 & 158.45 & 30.01 \\
Reatividade emocional & 33.07 & 11.36 & 37.44 & 11.18 & 30.82 & 10.8 \\
Posição do eu & 43.42 & 9.41 & 45.33 & 8.78 & 42.44 & 9.58 \\
Corte emocional & 49.61 & 10.2 & 48.75 & 9.43 & 50.07 & 10.55 \\
Fusão com os outros & 36.6 & 9.98 & 39.46 & 10.16 & 35.13 & 9.57 \\
\hline
\end{tabular}

A análise de variância (ANOVA) também sugeriu diferenças estatisticamente significativas entre as médias das variáveis diferenciação do self, considerando-se a faixa etária dos estudantes $[F(2,796)=5.35$; $p<.00]$. Os participantes mais velhos evidenciaram níveis mais elevados de diferenciação do self do que os mais novos, principalmente quando se comparam a primeira (18 a 23 anos) e a terceira faixa etária (28 a 35 anos). $\mathrm{O}$ mesmo ocorreu com as dimensóes: reatividade emocional $[\mathrm{F}(2,796)=4.41 ; \mathrm{p}<.01]$, posição do eu $[F(2,796)=3.21 ; p<.04]$ e 
fusão com os outros $[F(2,796)=7.71 ; p<.00]$. Já em relação à dimensão corte emocional, não foi observada diferença de média em função da faixa etária $[F(2,796)=2.26 ; p<.10]$.

A comparação entre as médias do escore geral da diferenciação do self em função do nível de satisfação com o curso foi estatisticamente significativa $[F(3,797)=6.66 ; p<.00]$, bem como das dimensões reatividade emocional $[F(3,797)=4.82 ; p<.02]$, posição do eu $[F(3$, $797)=4.99 ; p<.02]$ e corte emocional $[F(3,797)=5,34 ; p<.01]$, em que os estudantes muito satisfeitos com o curso apresentaram médias mais altas do que os insatisfeitos ou pouco satisfeitos. A única diferença não significativa foi relativa à dimensão fusão com os outros $[F(3$, 797) $=1.06 ; p<.36]$.

No que diz respeito aos resultados sobre funcionamento familiar, as médias e os desvios padrão de cada dimensão, gerais e por sexo, são evidenciados na Tabela 2. A fim de verificar possível associação entre o funcionamento familiar e o sexo dos universitários, foi realizado um teste qui-quadrado, que não demonstrou resultado estatisticamente significativo $\left(\chi^{2}=4.80 ; g l=5 ; p<.44\right)$.

\section{Tabela 2}

Funcionamento familiar: médias e desvios padrão gerais e por sexo

\begin{tabular}{lcccccc}
\hline & \multicolumn{2}{c}{ Geral } & \multicolumn{2}{c}{ Masculino } & \multicolumn{2}{c}{ Feminino } \\
\hline & $M$ & $D P$ & $M$ & $D P$ & $M$ & $D P$ \\
\hline Coesão equilibrada & 25.98 & 5.67 & 25.58 & 5.47 & 26.19 & 5.77 \\
Flexibilidade equilibrada & 26.5 & 6.23 & 26.29 & 6.31 & 26.61 & 6.20 \\
Coesão desengajada & 16.94 & 5.63 & 17.47 & 5.49 & 16.67 & 5.69 \\
Coesão emaranhada & 16.96 & 4.30 & 16.81 & 4.09 & 17.03 & 4.41 \\
Flexibilidade rígida & 17.33 & 5.03 & 17.43 & 4.65 & 17.28 & 5.23 \\
Flexibilidade caótica & 16.67 & 5.75 & 16.51 & 5.57 & 16.75 & 5.84 \\
Comunicação familiar & 32.98 & 8.72 & 33.07 & 8.58 & 32.93 & 8.81 \\
Satisfaçáo familiar & 32.14 & 8.76 & 32.26 & 8.47 & 32.08 & 8.92 \\
\hline
\end{tabular}


Para avaliar se havia diferenças de média das dimensóes do funcionamento familiar em função do sexo dos participantes, foi empregado o teste $\mathrm{t}$, que também não apresentou resultados significativos: coesão equilibrada $(t=-1.43 ; g l=797 ; p<.15)$; flexibilidade equilibrada $(t=-.68$; $g l=797 ; p<.49)$; coesão emaranhada $(t=-.68 ; g l=797 ; p<.49)$; flexibilidade rígida $(t=.39 ; g l=797 ; p<.14)$; flexibilidade caótica $(t=-.57 ; g l=797$; $p<.56)$; comunicação familiar $(t=.22 ; g l=797 ; p<.82)$; e satisfação familiar $(t=.27 ; g l=797 ; p<.78)$. Destaca-se que as mulheres apresentaram níveis mais elevados do que os homens apenas em relação à dimensão coesão desengajada $(t=1.90 ; g l=797 ; p<.05)$.

O teste qui-quadrado, empregado para avaliar se houve associação entre o funcionamento familiar e a faixa etária dos graduandos, resultou em tendência dos universitários mais velhos, da terceira faixa etária (entre 28 e 35 anos) a perceberem sua família dentro das tipologias consideradas mais saudáveis $\left(\chi^{2}=16.40 ; g l=10 ; p<.08\right)$. Já o resultado da ANOVA, relativo à comparação entre as médias do funcionamento familiar e da faixa etária, foi estatisticamente significativo somente para a dimensão coesão desengajada $[F(2,796)=3.45 ; p<.03]$. Os graduandos que se encontravam na terceira faixa etária apresentaram índices mais elevados de coesão desengajada do que os dois outros grupos. No que diz respeito às outras dimensóes, os resultados, não significativos, foram: coesão equilibrada $[F(2,796)=.93 ; p<.39]$; flexibilidade equilibrada $[F(2,760)=.84 ; p<.43]$; coesão emaranhada $[F(2,796)=.23 ; p<.78]$; flexibilidade rígida $[F(2,796)=1.43 ; p<.24]$; flexibilidade caótica $[F(2,796)=2.18 ; p<.11]$; comunicação familiar $[F(2,796)=.20 ; p<.81]$; e satisfação familiar $[F(2,796)=.46 ; p<.63]$.

$\mathrm{O}$ resultado da comparação das médias (ANOVA) do funcionamento familiar de acordo com a satisfação com o curso indicou resultado estatisticamente significativo para as seguintes dimensóes: coesão equilibrada $[F(3,797)=3.41 ; p<.01]$; flexibilidade equilibrada $[F(3,797)=4.32 ; p<.00]$; coesão desengajada $[F(3,797)=2.56 ; p<.05]$; e flexibilidade caótica $[F(3,797)=3.78 ; p<.01]$. Os graduandos insatisfeitos e poucos satisfeitos com o curso de graduação apontaram maiores níveis de coesão desengajada e flexibilidade caótica. Já os universitá- 
rios satisfeitos e muito satisfeitos apresentaram índices mais elevados nas duas dimensóes positivas do funcionamento familiar: coesão equilibrada e flexibilidade equilibrada. Portanto, percebe-se que os estudantes que avaliaram o funcionamento familiar de maneira mais positiva mostraram-se mais satisfeitos em relação ao curso.

Os dados que dizem respeito às correlaçóes entre as duas principais variáveis de interesse do estudo podem ser visualizados na Tabela 3. Observando-se o escore total da diferenciação do self e do funcionamento familiar, constata-se que houve correlação positiva entre os dois construtos, embora baixa $(r=.22 ; p<.05)$. A diferenciação do self também se correlacionou negativamente com as dimensóes coesão emaranhada $(r=-.22 ; p<.05)$ e positivamente com a satisfação familiar $(r=.25 ; p<.05)$ e com a comunicação familiar $(r=.20 ; p<.05)$.

Os resultados que dizem respeito às correlaçóes foram analisados de acordo com a premissa de que as correlaçóes entre .20 e .30 são consideradas baixas e as correlaçóes acima de .30 são moderadas (Field, 2009). Ao considerar todas as dimensóes das variáveis, a análise da Tabela 3 (Correlaçóes entre funcionamento familiar e diferenciação do self) permite constatar que a maior correlação encontrada foi entre o funcionamento familiar e a dimensão corte emocional da diferenciação do self $(r=-.42 ; p<.01)$. Destaca-se, ainda a correlação negativa moderada do corte emocional com a coesão desengajada ( $r=-.40$; $p<.01)$; e negativa baixa com a flexibilidade caótica $(r=-.28 ; p<.05)$. Embora em níveis menores, observa-se que o corte emocional também obteve correlação positiva com todas as dimensóes favoráveis do funcionamento familiar: coesão equilibrada $(r=.34 ; p<.01)$, flexibilidade equilibrada $(r=.30 ; p<.05)$, comunicação familiar $(r=.34 ; p<.01)$ e satisfação familiar $(r=.36 ; p<.01)$. A dimensão posição do eu apresentou correlaçóes positivas baixas com quase todas as dimensóes favoráveis do funcionamento familiar: satisfação familiar $(r=.22 ; p<.05)$, flexibilidade equilibrada $(r=.21 ; p<.05)$ e comunicação familiar $(r=.213 ; p<.05)$. Já a dimensão fusão com os outros obteve correlação negativa moderada com a coesão emaranhada $(r=-.30 ; p<.05)$ e baixa com a flexibilidade rígida $(r=-.23 ; p<.05)$. 


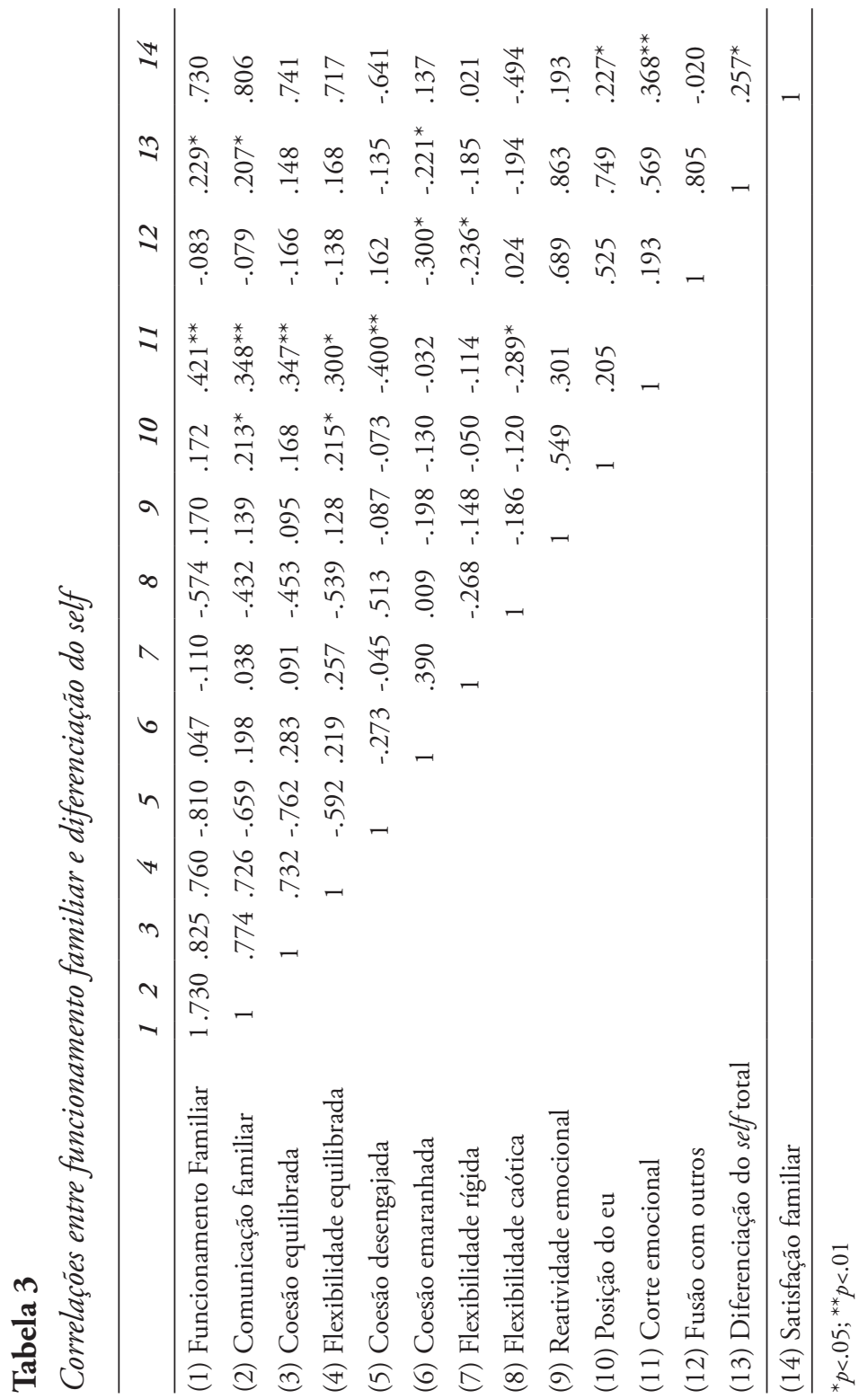




\section{Discussão}

O objetivo principal deste estudo foi analisar as relaçóes entre funcionamento familiar e diferenciação do self de jovens adultos universitários brasileiros. Os resultados encontrados permitem constatar que os estudantes com níveis mais elevados de diferenciação do self tendem a perceber positivamente a comunicação com a família e demonstrarem maior grau de satisfação com a relação familiar, em consonância com os resultados anteriormente obtidos por Kim et al. (2014) e Kim et al. (2015). Além disso, esses graduandos, com níveis maiores de diferenciação, também enquadraram suas famílias dentro de tipologias caracterizadas pelo equilíbrio entre coesão e flexibilidade, ou seja, que fornecem apoio emocional e mostram-se mais adaptáveis diante de fases de transição, legitimando os achados de investigaçóes anteriores (Chung \& Gale, 2008; Lee \& Cha, 2000; Lee \& Lee, 1997).

Destacam-se algumas relaçóes mais expressivas encontradas, por exemplo, entre funcionamento familiar e as dimensóes corte emocional e posição do eu, indicando que a capacidade do jovem adulto de se posicionar diante da família, expressando suas expectativas; aliada ao estabelecimento de regras e limites claros no âmbito familiar, tende a facilitar o processo de diferenciação. Atenta-se, ainda, para a relação obtida entre as dimensões favoráveis ao funcionamento saudável da família (coesão equilibrada e flexibilidade equilibrada) com o corte emocional, sugerindo que certo distanciamento emocional em relação à família de origem pode ser positivo durante a fase da adultez jovem/ acadêmica. A integração desses achados parece corroborar os pressupostos de Bowen (1978) e Minuchin (1982) e os achados de Pellegrini et al. (2015). Segundo esses autores, quando há envolvimento excessivo dos pais, sem definição clara de papéis, funçóes e hierarquias, as famílias tendem a apresentar dificuldade para lidar com as crises geradas pelas transiçóes do ciclo vital e o processo de diferenciação dos filhos acaba sendo prejudicado.

Os achados que tratam da interface entre os dois principais construtos explorados nesta investigação _ diferenciação do self e fun- 
cionamento familiar _ e o nível de satisfação com o curso também são bastante pertinentes, tendo em vista a influência dessa satisfação na capacidade de enfrentamento das responsabilidades inerentes à universidade por parte dos jovens adultos, e, por consequência, na possiblidade de evasão. Ressalta-se que a insatisfação com o curso foi mais frequente nas famílias rígidas, ou seja, com maior desligamento emocional e menor nível de flexibilidade para lidar com mudanças. Parece, desse modo, que quando as famílias conseguem ser flexíveis e comunicativas durante o período acadêmico, os graduandos mostram-se mais satisfeitos com sua vivência no curso. Esse resultado é interessante, ao mostrar a importância do funcionamento e da comunicação familiar também em termos de resultados acadêmicos, já indicada anteriormente na literatura (Guan et al., 2016; Slaten \& Baskin, 2014).

No que diz respeito aos resultados sobre o sexo dos participantes, o fato das universitárias avaliarem com maior frequência o padrão de sua família como distante, além de apresentarem níveis menores de diferenciação do que os graduandos, pode representar um conflito maior para as mulheres em relação aos desafios intrínsecos ao período da adultez jovem e acadêmico. Propondo-se uma reflexão cultural acerca desses dados, elementos de ordem profissional nem sempre ocupam um lugar prioritário na vivência das jovens adultas. Essa premissa pode ser explicada por dois motivos: os homens geralmente são educados para assumir um papel na sociedade brasileira mais fortemente ligado ao desempenho e ao sucesso profissional; e apesar da diminuição progressiva das diferenças de gênero na realidade nacional, as jovens adultas ainda são socialmente exigidas de forma diversificada, dividindo as questôes de carreira com a escolha de um parceiro e a opçáo pela maternidade (Lassance \& Magalhães, 1997). Portanto, supóe-se que esses fatores imponham ao público masculino a necessidade de um processo de diferenciação mais rápido se comparados às mulheres.

Os resultados referentes à idade dos participantes são condizentes com os encontrados por Kim et al. (2014), que identificaram níveis maiores de diferenciação entre participantes mais velhos. Neste estudo, os graduandos da terceira faixa etária (entre 28 e 35 anos) também apre- 
sentaram uma percepção mais saudável do funcionamento familiar, se comparados aos mais novos, e maiores níveis de coesão desengajada, que sugere menor envolvimento emocional com a família de origem. A integração desses dados pode ser interpretada a partir das premissas defendidas por Aylmer (1995), e, Carter e McGoldrick (1995). Ambos os autores argumentam que a adultez jovem é normalmente demarcada pela remodelagem e ressignificação das relações entre pais e filhos, de modo que os pais tentam estabelecer um relacionamento menos hierárquico e os jovens adultos buscam pela aquisição de autonomia.

Refletindo-se acerca desses dados e ampliando-se a discussão para o período de graduação, supóe-se que embora o suporte dos pais seja crucial durante a fase universitária, o envolvimento em excesso pode influenciar negativamente na diferenciação. Esse impacto possivelmente também venha a ser sentido por meio da satisfação com o curso, que reflete, dentre outros fatores, a forma como o jovem adulto enfrenta os desafios da vivência acadêmica.

\section{Consideraçóes Finais}

Ao avaliar os principais resultados obtidos por meio desta pesquisa sob o ponto de vista da perspectiva familiar sistêmica, observa-se que as famílias consideradas emaranhadas, ou seja, que não possuem clareza entre os papéis dos membros e são caraterizadas pelo envolvimento emocional excessivo, parecem ser as mais prejudiciais ao processo de diferenciação do self dos jovens adultos graduandos.

Dentre as principais contribuiçóes deste estudo, destaca-se a importância do equilíbrio entre controle/apoio e estímulo da família para a aquisição de independência e autonomia por parte dos jovens adultos, durante o período universitário. Essa constatação pode servir como aporte para a prática de psicólogos com estudantes de graduação, seja em terapia individual/familiar ou em processos de aconselhamento de carreira. Uma das principais tarefas esperadas durante a adultez jovem trata-se do estabelecimento e da concretizaçáo de objetivos pro- 
fissionais. Portanto, o diagnóstico e as intervençóes profissionais com esse público requerem uma integração das informaçóes sobre a percepção dos jovens adultos acerca de sua vivência acadêmica e o padrão de funcionamento da família, que pode repercutir positiva ou negativamente no processo de diferenciação e também na satisfação com o curso de graduação.

Os resultados relacionados à satisfação com o curso podem colaborar para que as áreas de gestão universitária implementem açóes que envolvam a família dos estudantes, como, por exemplo, a promoção de um momento de integração na entrada à universidade, para que a família se aproprie desse espaço e adquira conhecimentos sobre os futuros desafios de seus filhos. Outra prática, de natureza preventiva, refere-se à parceria da universidade com escolas de ensino médio, a fim de viabilizar workshops e palestras sobre temáticas de orientação profissional, envolvendo pais e alunos.

Ressalta-se que os achados obtidos por meio deste estudo não podem ser generalizados para todo o público universitário do Brasil, tendo em vista que o País é reconhecido pela grande diversidade social, econômica e cultural. Ao analisar que a maioria dos estudantes são naturais da regiáo sul, pontua-se que os Estados dos quais fazem parte destacam-se positivamente nos índices socioeconômicos e são perpassados por singularidades, que, apesar de não exploradas nesta investigação, precisam ser ponderadas.

Como limitaçôes desta pesquisa, verifica-se que algumas correlaçóes encontradas entre as variáveis de interesse foram fracas ou moderadas. Embora isso não represente um problema quando se consideram fenômenos psicológicos, é importante observar que esta investigação não confere relaçóes de causalidade entre as variáveis. Além disso, o fato da escala FACES IV ainda não possuir validação completa no contexto brasileiro também precisa ser assinalado, apesar dos bons índices de consistência interna obtidos neste estudo.

Conforme já explicitado, pesquisas que exploram a relação entre funcionamento familiar e diferenciação do self de jovens adultos el ou universitários ainda são escassas, principalmente no Brasil. Dessa 
forma, a temática apresenta amplas possibilidades de investigação científica, por meio do emprego de métodos qualitativos, mistos e longitudinais, e não apenas quantitativos. A integração dos construtos com outras variáveis que não foram abordadas neste estudo, como o nível socioeconômico e educacional da família, também poderia trazer outros elementos para a melhor compreensão da interface entre funcionamento familiar e diferenciação do self. Por fim, a investigação da relação entre aspectos familiares, processo de diferenciação e fatores mais específicos da vivência universitária _ como ajustamento acadêmico, saúde psicológica dos graduandos e desempenho acadêmico _ também merece aprofundamento, tendo a vista a necessidade de adaptação, tanto do jovem adulto quanto de sua família, aos desafios da entrada e permanência no universo acadêmico.

\section{Referências}

Ambiel, R., Hernández, D. N., \& Martins, G. H. (2016). Relações entre adaptabilidade de carreira e vivências acadêmicas no ensino superior. Psicología desde el Caribe, 33(2), 158-168. http://dx.doi. org/10.14482/psdc.33.2.7071

Aylmer, R. C. (1995). O lançamento do jovem adulto solteiro. In: Carter, B. \& McGoldrick, M. (1995). As mudanças no ciclo de vida familiar: uma estrutura para a terapia familiar (pp. 169-183). (M. A. V. Veronese, Trad.). 2 ed. Porto.

Bardagi, M., \& Albanaes, P. (2015). Relaçóes entre Adaptabilidade de carreira e personalidade: Um estudo com universitários ingresantes. Revista PSICOLOGIA, 29(1), 35-44. https:/doi. org/10.17575/rpsicol.v29i1.989

Bardagi, M. P., \& Hutz, C. S. (2010). Satisfação de vida, comprometimento com a carreira e exploraçáo vocacional em estudantes universitários. Arquivos Brasileiros de Psicologia, 62(1), 159-170. 
Böing, E. (2014). Relaçôes entre coparentalidade, funcionamento familiar e estilos parentais em uma perspectiva intergeracional. Teses de doutorado. Universidade Federal de Santa Catarina.

Bowen, M. (1978). Family Therapy in clinical practice. New York: Jason Arons.

Bueno, R. K., Souza, S. A., Monteiro, M. A., \& Teixeira, R. H. M. (2013). Processo de diferenciação dos casais de suas famílias de origem. Psico, 44(1), 16-25.

Carter, B., \& McGoldrick, M. (1995). As mudanças no ciclo de vida familiar: uma estrutura para a terapia familiar (M. A. V. Veronese, Trad.). 2 ed. Porto Alegre: Artes Médicas. (Original publicado em 1989).

Guan, P., Capezio, A., Restubog, S. L. D., Read, S., Lajomb, J. A. L., $\& \mathrm{Li}, \mathrm{M}$. (2016). The role of traditionality in the relationships among parental support, career decision-making self-efficacy and career adaptability. Journal of Vocational Behavior, 94, 114-123. https://doi.org/10.1016/j.jvb.2016.02.018

Cerveny, C. M. O. (1994). A família como modelo: desconstruindo a patologia. Campinas: Editorial Psy II.

Chung, H. J., \& Gale, J. (2008). Family functioning and selfdifferentiation: A cross-cultural examination. Contemporary Family Therapy, 31, 19-33. https://doi.org/10.1007/ s10591-008-9080-4

Field, A. (2009). Descobrindo a estatística usando o SPSS. 2. ed. Porto Alegre: Artmed.

Johnson, P., Thorngren, J., \& Smith, A. (2001). Parental divorce and family functioning: Effects on differentiation levels of young adults. The Family Journal, 9(3), 265-272. https://doi. org/10.1177/1066480701093005

Kim, H., Prouty, A. M., Smith, D B., Ko, M., Wetchler, J. L., \& Oh, J. (2014). Differentiation of self and its relationship with family functioning in South Koreans. The American Journal of Family Therapy, 4, 257-265. https://doi.org/10.1080/01926187.2013.83 8928 
Kim, H., Prouty, A. M., Smith, D. B., Ko, M., Wetchler, J. L., \& Oh, J. (2015). Differentiation and healthy family functioning of Koreans in south Korea, south Koreans in the united states, and white Americans. Journal of Marital and Family Therapy, 41(1) 72-85. http://doi/10.1111/jmft.12049

Lassance, M.C.P., \& Magalhães, M.O. (1997). Gênero e escolha profissional. In: R.S. Levenfus (Org.). A Psicodinâmica da escolha profissional (pp. 47-61). Porto Alegre: Artes Médicas.

Lee, S., \& Cha, Y. E. (2000). A study on the effects of adult children's differentiation of self and the healthy family-of-origin on the solidarity with aged mother. Journal of the Korean Home Economics Association, 38(4), 21-32.

Lee, C., \& Lee, H. (1997). Family system types, self-differentiation and psychological well-being of adolescent. Journal of the Korean Home Economics Association, 35(3), 101-113.

Major, S., Rodríguez González, M., Miranda, C., Rousselot, M., \& Relvas, A (2014). Inventário de diferenciação do Self-Revisto (IDS-R). Avaliação Familiar: funcionamento e intervenção, 1, 71-96. Imprensa da Universidade de Coimbra. http://dx.doi. org/10.14195/978-989-26-0839-6_3

Martins, E. M. A., Rabinovich, E. P., \& Silva, C. N. (2008). Família e processo de diferenciação na perspectiva de Murray Bowen: Um estudo de caso. Psicologia USP, 19(2), 181-197. https://doi. org/10.1590/S0103-65642008000200005

Miller, R. B., Anderson, S., \& Keala, D. K. (2004). Is Bowen Thoery valid? A review of basic research. Journal of Marital and Family Therapy, 30(4), 453-466. http://doi/10.1111/j.1752-0606.2004. tb01255.x

Minetto, M. de F. J. (2010). Práticas educativas parentais, crenças parentais, estresse parental e funcionamento familiar de pais de crianças com desenvolvimento típico e atípico. Tese de doutorado. Universidade Federal de Santa Catarina.

Minuchin, S. (1982). Famílias: Funcionamento e tratamento. Porto Alegre: Artmed. 
Olson, D.H., \& Gorall, D. M. (2006). FACES IV \& the Circumplex Model.

Olson, D. H., Gorall, D. M., \& Tiesel, J, W. (2007). FACES IV \& the Circumplex Model: Validation study.

Pellegrini, P. G., Silva, I. M., Barreto, M., \& Crepaldi, M. A. (2015). Diferenciaçáo do adulto jovem: um estudo de caso em atendimento familiar. Pensando Famílias, 19(1), 114-129.

Reis, L. P. C., \& Rabinovich, E. P. (2006). O fantasma da repetição e a relação mãe / filha. Revista Brasileira de Crescimento e Desenvolvimento Humano, 16(3), 39-52.

Skowron, E. A., \& Friedlander, M. L. (1998). The Differentiation of Self Inventory: development and initial validation. Journal of Counseling Psychology, 45, 235- 246. http://doi/10.1037/ a0016709

Skowron, D. A., \& Schmitt, T. A. (2003). Assessing interpersonal fusion: Reliability and validity of a new DSI Fusion with Others Scale. Journal of Marital and Family Therapy, 209-220. http:// doi/10.1111/j.1752-0606.2003.tb01201.x

Slaten, C. D., \& Baskin, T. W. (2014). Examining the impact of peer and Family belongingness on the career decision-making difficulties of young adults: a path analytic approach. Journal of Career Assessment, 22, 59-74. http://doi/10.1177/1069072713487857

Recibido: 2019-01-31

Revisado: 2021-03-13

Aceptado: 2021-04-08 